\title{
Bei der Sterbehilfe sind die Ärzte vierfach gefordert
}

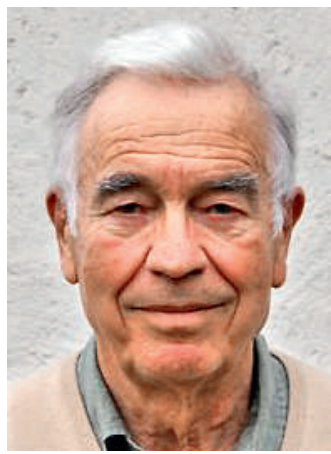

Hans Stalder
Beihilfe zum Suizid ist in der Schweiz straflos, sofern sie nicht aus selbstsüchtigen Beweggründen erfolgt. Dies gilt für jedermann, also auch für Ärzte. Letztere spielen aber - ob sie wollen oder nicht - eine wichtige Rolle in der Suizidhilfe, und dabei sind sie vierfach gefordert.

\section{Eine zugrundeliegende Depression ausschliessen}

Der Wunsch, sich das Leben zu nehmen, kann ein Symptom für eine schwere Depression sein, die potentiell behandelbar und reversibel ist. Daher ist es die Pflicht des Arztes, diese Erkrankung abzuklären und wenn möglich zu behandeln oder die Person an einen Spezialisten zu überweisen, um eine Verzweiflungstat zu verhindern, worüber der Betroffene im Nachhinein vielleicht froh wäre. Diese Pflicht erstreckt sich auf andere potentiell heilbare Symptome, wie Schmerz oder Dyspnoe, die einer Behandlung bedürfen.

1 Betreuung von Patientinnen und Patienten am Lebensende. 2004. Medizinisch-ethische Richtlinien der Schweizerischen Akademie der Medizinischen Wissenschaften

2 Stalder H. Ethik ja aber bitte ohne Belehrung! Schweiz Ärztezeitung. 2012;93(27/28):1072.

3 Schafroth M. Suizidhilfe ist Teil der ärztlichen Aufgaben - die Zeit ist reif für eine Anpassung der SAMW-Richtlinien. Schweiz Ärztezeitung. 2012;93(34):1226-7.

4 Mauron A. Assistance au suicide, un chantier éthique perpétuel. Le Temps. 31. Mai 2013.

5 www.ejpd.admin.ch

6 Zentrale Ethikkommission der Schweizerischen Akademie der Medizinischen Wissenschaften. Probleme bei der Durchführung von ärztlicher Suizidhilfe. Schweiz Ärztezeitung. 2012;93(11):411-2.

* Prof. Dr. med. Hans Stalder, Facharzt für Innere Medizin, Redaktionsmitglied, ehemaliger Chefarzt der Policlinique de Médecine und des Département de Médecine communautaire des Hôpitaux Universitaires de Genève

hans.stalder[at]saez.ch

\section{Eine unmittelbar zum Tode führende Erkrankung diag-} nostizieren

Diese Forderung ist in den Richtlinien der Schweizerischen Akademie der Medizinischen Wissenschaften (SAMW) über das Verhalten von Ärzten gegenüber dem Wunsch nach Suizid [1] festgehalten und wurde so auch in die Standesordnung der FMH übernommen. Über diese Forderung wird zurzeit debattiert $[2,3]$.

\section{Die Urteilsfähigkeit der Person beurteilen}

Suizid ist ein freiwilliger Akt, und der Sterbewillige muss bei vollem Bewusstsein und urteilsfähig sein. Diese Bedingung verbietet jegliche Sterbehilfe bei Patienten, die diese Fähigkeit nicht mehr haben, auch wenn der Wunsch in einer Patientenverfügung festgehalten ist. Solche Verfügungen sollen ermöglichen, nicht erwünschte medizinische Massnahmen $\mathrm{zu}$ verhindern, erlauben aber nicht, ärztliche Eingriffe wie Beihilfe zum Suizid zu fordern.

4. Das tödliche Medikament verordnen und verabreichen Der Gesetzgeber spricht nicht über die Mittel, die zum Tod des Sterbewilligen führen. Diese sollten aber menschlich und so schmerzfrei wie möglich sein. Dies ist zurzeit nur durch ein ärztliches Rezept möglich. Ihre Verabreichung wird heute meist durch dere durchgeführt.

Und schliesslich haben Ärzte noch eine fünfte - administrative - Aufgabe zu erfüllen: Sie müssen den Tod feststellen. Der Tod infolge von Beihilfe zum Suizid muss wie alle anderen unnatürlichen Todesfälle «Sterbehilfespezialisten» wie EXIT, Dignitas und an- den Behörden gemeldet werden, was nur durch einen Arzt erfolgen kann.

Zu ergänzen wäre noch, dass beim Wunsch nach Suizidbeihilfe die Aufgabe des Arztes vermutlich weniger darin bestehen sollte, Diagnosen zu stellen, als vielmehr zu beurteilen, ob der Entschluss der Person selbstbestimmt, wohlüberlegt und definitiv ist [4].

Gemäss dem Vorschlag des Eidgenössischen Polizei- und Justizdepartements von 2009 [5], der aufgrund fehlender Unterstützung in der Vernehmlassung zurückgezogen wurde, hätten die unter 2, 3 und 4 erwähnten Massnahmen von drei verschiedenen Ärzten vorgenommen werden müssen. Die SAMW sprach sich damals vehement dagegen aus, den Ärzten in der Beihilfe zum Suizid eine solch zentrale Rolle zuzuweisen [6]. Ihrer Ansicht nach widerspricht die Sterbehilfe der medizinischen Ethik und gehört nur bedingt zu den ärztlichen Aufgaben [1]. Wer aber könnte diese Hilfe leisten, wenn nicht die Ärzte? Punkt 1 bis 3 betreffen medizinische Diagnosen, Punkt 4 die nur dem Arzt vorbehaltene Rezeptierung, da ein freier Verkauf des Medikaments in der Apotheke kaum denkbar ist. Und schliesslich kann nur ein Arzt den Tod feststellen.

Infolge eines Urteils des Europäischen Gerichtshofs für Menschenrechte in Strassburg wurde der Gesetzgeber erneut angerufen, die Beihilfe zum Suizid gesetzlich zu regeln (die Schweiz hat gegen dieses Urteil Berufung eingelegt).

Meiner Ansicht nach sollte der unwiderrufliche Wunsch, das eigene Leben zu beenden, weder durch gesetzliche Hürden noch durch Beiziehung von fremdem Personal erschwert werden. Somit sollten idealerweise die vier Schritte von ein und demselben, den Betroffenen persönlich behandelnden Arzt vorgenommen werden (wobei er dazu selbstverständlich nicht verpflichtet werden kann). Mir ist bewusst, dass diese Einstellung weder der heutigen noch der tausendjährigen Standesethik der Ärzte entspricht. Aber die Zeiten ändern sich ... Ist es nicht unsere Aufgabe als Ärzte, darüber nachzudenken oder aber zumindest dafür Sorge zu tragen, dass ein neues Gesetz diesen so schwierigen Moment des Lebens nicht in einer Flut administrativer Auflagen erstickt? Vergessen wir nicht, dass die aktuelle Schweizer Gesetzgebung grossen Spielraum zulässt!

Hans Stalder* 